\title{
Costs analysis of Staged Versus Simultaneous Bilateral Total Knee and Hip Arthroplasty囚a university affiliated hospital survey of 1579 Chinese patients
}

Ji-Fei Hou

Qingdao University Medical College

Chuan $\mathrm{Hu}$

Qingdao University Medical College

Yan-Zheng Liu

Shandong University Qilu Hospital

Chi Zhang

Qingdao University Medical College

Jing Li

Affiliated Hospital of Qingdao University

Yue Wang

Qingdao University Medical College

Cheng-Cheng Li

Guangxi Medical University

Yun Zhang ( $\sim 18186532881 @ 163 . c 0 m$ )

https://orcid.org/0000-0002-1358-6667

\section{Research article}

Keywords: Total knee arthroplasty, Total hip arthroplasty, Costs, Comparison

Posted Date: August 13th, 2019

DOI: https://doi.org/10.21203/rs.2.12776/v1

License: () This work is licensed under a Creative Commons Attribution 4.0 International License. Read Full License 


\section{Abstract}

Background Total knee arthroplasty (TKA) and total hip arthroplasty (THA) are required for many patients. No recent studies that concentrate on the costs for each of the payment items both for TKA and THA when comparing simultaneous bilateral procedures with staged arthroplasty. This study aimed to evaluate the medical costs, length of stay(LOS), blood transfusion, and in-hospital complications in patients undergoing simultaneous TKA or THA and staged TKA or THA. Methods A retrospective cohort study was conducted by analyzing 1,579 patients from a single institution. All patients who underwent primary bilateral TKA or THA from 2013 to 2018 were divided into simultaneous bilateral and staged bilateral groups. These two sub-groups were compared between three groups(TKA, THA, and pooled groups). Results All categories of medical costs, except for materials fees which were higher in the simultaneous bilateral THA and pooled groups but with no statistical significance, were lower in the simultaneous TKA, THA and pooled simultaneous groups. The total average medical costs in simultaneous and staged bilateral TKA groups were $\$ 15,535.621$ (\$12,362.542,\$16,450.131)and $\$ 16,747.192(\$ 14,670.273, \$ 18,036.289),(p<0.001)$, respectively; THA groups were $\$ 15,046.210$ (\$12,838.230, $\$ 17,026.529)$ and $\$ 16,126.808$ (\$14,575.031, $\$ 18,867.963),(p=0.003)$, respectively; pooled groups were $\$ 16,506.971$ (\$14,618.267, $\$ 18,185.414)$ and $\$ 15,467.561(\$ 12,432.783, \$ 16,494.155),(p<0.001)$, respectively. The highest and lowest costs were materials fees and nursing care fees. No significant differences were found for complications between the two sub-groups in three groups. The simultaneous groups had a shorter LOS, a higher incidence of blood transfusion than staged groups whether it is TKA groups or THA or pooled groups. Conclusions These results indicate that simultaneous bilateral TKA and THA with a shorter LOS is more cost-effective than staged bilateral TKA and THA. Counts of complications were not affected by the choice for staged or simultaneous bilateral total arthroplasty. But blood transfusion was more prevalent in the simultaneous groups both for TKA and THA.

\section{Background}

Total Knee Arthroplasty (TKA) and Total Hip arthroplasty (THA) are cost-effective procedures for patients with advanced arthropathies, such as osteoarthritis (OA), rheumatoid arthritis (RA), and hip fracture, which can improve the quality of life for these patients significantly [1-3]. With the aging population, arthropathy incidence is projected to continue growing in many countries[2,4-6].

Besides, the rising number of arthroplasty procedures, corresponding with an increased yearly cost, will result in a huge economic burden for both the families and the health care system[2,7-10]. As China's healthcare expenditures are soaring[11], the government has sought to reduce the overall cost of arthroplasty procedures by shortening average LOS, strengthening the supervision of surgical procedures and management of purchasing medical disposable materials.

However, there exists debate regarding performing staged bilateral TKA or THA with an interval between procedures or simultaneous bilateral TKA or THA patients, primarily because of the controversy about the outcomes of two procedures[7,12]. Therefore, the surgeons and patients need some new evidence to decide whether to perform simultaneous bilateral TKA (or THA) or staged TKA (or THA).

Previous studies about simultaneous bilateral TKA versus staged TKA and simultaneous THA vs staged THA have made some progress. But their research focuses always concentered on clinical outcomes, such as operative and anesthetic times, EuroQol-5D index, health-related quality of life, blood loss, Harris hip score, and in-hospital mortality rates[1,1215]. Whereas to the best of our knowledge, no recent study has presented a direct comparison of actual hospital costs for both TKA and THA. Yet there is no consensus on which procedure(simultaneous vs staged bilateral TKA or THA) is better.

In our research, we sought to provide data on this issue by analyzing 1,579 patients referred to our hospital. This study aimed to investigate differences in cost, in-hospital complications, blood transfusion, and length of stay in three groups(TKA, THA, and pooled patients groups), respectively. Besides, subgroup analysis of cost, in-hospital complications, 
blood transfusion, and length of stay were performed in staged and simultaneous bilateral total joint arthroplasty(TJA) groups.

\section{Methods}

\section{Study Design and Data Sources}

After Ethics Committee of the Affiliated Hospital of Qingdao University approval, we performed a retrospective analysis of patients who underwent primary TKA or THA procedures discharged from January 1th, 2013, to December 31th, 2018, referred to the Affiliated Hospital of Qingdao University, a 5046-bed urban, third grade class A, medical-school-affiliated hospital in Qingdao, Shandong(third grand class A is the highest level in the hospital classification in China).

Anonymous clinical data were extracted from the hospital information system (HIS) of our hospital, which were consisted of baseline information of the patient, in-hospital costs, LOS, indications of operation by International Classification of Diseases,10th revision (ICD-10), in-hospital complications, blood transfusion, and detailed healthcare expenditure information.

\section{Sample of Patients Selection}

Patient who underwent two procedures separated within 1 year and by the same surgeon was considered to have undergone staged bilateral TKA or THA. Patients were included in the analysis if all cost, in-hospital complication, LOS and demographic data were available.We excluded patients who underwent revision arthroplasty. We also excluded patients who underwent TJA of both sides during one hospitalization and infectious joint disease, as those patients would cost more. Patients were divided into three groups-THA, TKA, and all patients, and then divided into two subgroups according to whether they were undergoing simultaneous bilateral TJA or staged bilateral TJA.

\section{Primary Outcome: Direct Medical Costs}

Total medical expenses were mainly composed of bed fees, fees of general therapy, nursing care fees, check-up and laboratory test fees, surgical fees, drug fees, materials fees and charge for medical service. Total hospital costs of the staged group used pooled data of the two procedures. To offset the influence of inflation and economic growth and to reflect the real dollar value, all expenditure variables in the present study were adjusted to 2019 Chinese currency values using the Consumer Price Index (CPI), and then all values were converted to US Dollars (USD) with the exchange rate of 1 USD = 6.51 yuan (the averaged exchange rate from 2013 to 2018).

\section{Secondary Outcomes: In-Hospital Complications, Blood Transfusion, and LOS}

One of the secondary outcomes of interest was in-hospital complications (including acute myocardial infarction, deep venous thrombosis, pulmonary embolism, ileus, renal failure, pneumonia, and orthopedic-specific complications). Other secondary outcomes of interest included perioperative transfusion rate, and LOS which was defined as the duration of hospital admission before and after surgery.

\section{Statistical Analysis}


The normality of continuous variables was assessed using the Kolmogorov-Smirnov test or Shapiro-Wilk test. Nonnormally distributed variables were presented as medians and interquartile ranges (IQR, the range between the $25^{\text {th }}$ and $75^{\text {th }}$ percentile) and categorical variables were presented by counts and percentages. Demographic and clinical data of patients were compared between groups with the chi-squared test for categorical variables and the Mann-Whitney $U$ test for continuous variables. Atwo-sided $p<0.05$ was considered as statistical significance. All statistical analyses were performed using SPSS software version 25.0(IBM Corporation, USA).

\section{Results}

\section{Baseline Characteristics}

The baseline characteristics were shown in Table 1(tables at the end of the text). A total of 1,180 patients underwent TKA, of which 789 were simultaneous TKA patients and 391 were staged THA patients. Simultaneous TKA patients were younger $(64[60,69]$ vs $68[63,73]$ years, $p<0.001)$, with a higher incidence of blood transfusion(302, yes [38.28\%] vs 32 , yes [8.18\%], $p<0.000)$, and had a shorter LOS $(9[8,11]$ vs $18[15,20], p<0.001)$ compared with staged THA patients. For the distribution of gender, $82.51 \%$ were female for simultaneous TKA patients and $80.31 \%$ for staged THA patients. But no statistical significance between the two groups $(p=0.356)$. And no significant difference was found for complications either(20, yes [2.53\%] vs 11 , yes [2.81\%], $p=0.778)$.

399 patients received THA, consisting of 140 who underwent simultaneous bilateral THA and 259 staged THA. The patients of staged bilateral THA were older $(58[49,64]$ vs $53[49,64]$ years, $p<0.01)$, had a lower incidence of blood transfusion(36, yes [13.90\%] vs 51 , yes [36.43\%], $p<0.000)$, and with a longer LOS $(16[14,18]$ vs $10[8,11]$ years, $p<0.001)$ than those simultaneous THA patients. The gender distribution was neither different (42, female [30.00\%] vs 85 , female [32.82\%], $p=0.564$ ) between simultaneous THA patients and staged bilateral THA patients, nor was the count of complications $(4$, yes [2.86\%] vs 4 , yes [1.54\%], $p=0.564)$. As shown in Table 1.

Between 2013 and 2018, a total of 8,760 patients underwent arthroplasty in our hospital, and 1,579 patients were enrolled in this study. Among them, 929 cases were simultaneous total arthroplasty patients, and 650 cases were staged bilateral total arthroplasty patients. Similar to trends for TKA and THA, simultaneous total arthroplasty were performed more frequently in younger patients $(63[58,68]$ vs $64[58,70]$ years, $p<0.05)$, with a higher incidence of blood transfusion(353, yes $[37.99 \%]$ vs 68 , yes $[10.46 \%], p<0.000)$, and had a shorter LOS $(9[8,11]$ vs $17[15,19]$ years, $p<0.001)$. No statistical significance was found for complications (24, yes [2.58\%] vs 15 , yes [2.31\%], $p=0.728$ ). For patients pooled together, there was a difference in gender distribution $(693$, female [74.60\%] vs 399 , female $[61.38 \%], p<0.01)$ between simultaneous total arthroplasty patients and staged bilateral total arthroplasty patients(Table 1).

\section{Patient Expenditures}

The medical costs for each of the payment items were significantly different (all, $p<0.001$ ), and all costs were lower in the simultaneous bilateral TKA group than in the staged bilateral TKA group. As shown in Table 2. The total average medical expenses of simultaneous TKA was $\$ 15,535.621(\$ 12,362.542, \$ 16,450.131)$ and staged TKA was $\$ 16,747.192(\$ 14,670.273, \$ 18,036.289)$, with the difference value of $\$ 1,211.571$. For other payment items of medical costs, the highest cost was materials fees $(\$ 12,037.664[\$ 9,439.459, \$ 13,399.542]$ vs $\$ 12,245.999[\$ 9,796.633$, $\$ 12,702.510])$ and the lowest cost was nursing care fees $(\$ 59.908[\$ 34.869, \$ 73.733]$ vs $\$ 88.479[\$ 60.829, \$ 110.599])$ (Table 1).

The medical costs for almost all payment items of patients, except for materials fees $(\$ 11,794.072[\$ 9,676.080$, $\$ 14,000.301]$ vs $\$ 11,454.094$ [\$10,212.994, $\$ 13,680.321], p=0.441)$, were significantly difference in two sub-group of 
THA. As a whole, the total average medical cost of patients who underwent a simultaneous bilateral THA was significantly lower than in those who received a staged bilateral THA $(\$ 15,046.210[\$ 12,838.230, \$ 17,026.529]$ vs $\$ 16,126.808$ $[\$ 14,575.031, \$ 18,867.963], p=0.003)$, with the difference value of $\$ 1,080.598$. The highest and the lowest cost were also materials fees and nursing care fees $(\$ 59.908[\$ 33.180, \$ 73.733]$ vs $\$ 96.774[\$ 66.359, \$ 119.816], p<0.001)$ respectively(Table 1).

In general, the same results were presented by combining all patients who received TKA and THA together. As shown in Table 2. Costs of bed fees, fees of general therapy, nursing care fees, check-up, and laboratory test fees, surgical fees, charge for medical service, and drug fees were significantly lower compared with their counterparts (simultaneous bilateral total arthroplasty vs staged bilateral arthroplasty, all, $p<0.001)$. The comparison among materials fees has a different result with no statistical significance $(\$ 11,860.321[\$ 9,485.591, \$ 13,418.210]$ vs $\$ 11,707.530[\$ 9,928.480$, $\$ 12,846.894], p=0.736)$. Moreover, the total medical cost was significantly higher in patients who received a staged bilateral total arthroplasty than in those who underwent a simultaneous bilateral total arthroplasty $(\$ 16,506.971$ $[\$ 14,618.267, \$ 18,185.414]$ vs $\$ 15,467.561$ [\$12,432.783, \$16,494.155]). Just like TKA and THA, the same trend for the highest and the lowest costs, they were materials fees and nursing care fees $(\$ 59.908$ [\$34.716, $\$ 73.733]$ vs $\$ 92.166$ [\$64.516, \$115.207]) respectively(Table 1).

\section{Discussion}

Despite bilateral knee and hip arthroplasty being frequently performed, most studies paid attention to clinic outcomes, and only a few studies have been published recently on this topic. However, previous studies have not compared costs about THA, to say nothing of combining them[2,7,12]. In this study, we compared the cost of simultaneous and staged procedures among TKA, THA, and their combination, found a significant difference between the two groups. Viewing the general conclusions as a whole, the results showed that almost all kinds of costs were higher in staged TKA, THA and combination simultaneous group.

Our results are consistent with previous studies, which have estimated the cost-effectiveness of simultaneous TKA or THA, compared with staged TKA or THA [2,16-19]. A study from Taiwan demonstrated that all categories of medical costs, except for therapeutic procedure fees, were lower in the simultaneous TKA group[2]. However, a recent single-center study concluded that there was no significant difference in total hospital costs between two groups of TKA, and it might be explained that the majority of their patients who received simultaneous TKA underwent patient rehabilitation(IPR) unit admission, and IPR costs were added for all patients discharged to IPR[7].

At the same time, many studies including our study, which proved the cost-effectiveness of simultaneous TKA or THA, have found a shorter LOS in simultaneous TKA or THA. Compared with simultaneous surgery, staged surgery will increase the number of anesthetics and hospital admissions, which usually means a longer LOS which has been regarded as a pivotal indicator of hospital efficiency and quality of health care[20]. Prolonged LOS will not only have a negative impact on health outcomes, causing iatrogenic illness easily, but also high hospital occupancy resulted in a resultant loss of efficiency and access, all of which would bring a marked increase in health expenses[21,22]. And this might be explained the relationship between exceeding LOS with high cost, for long LOS unusually followed high costs[21-23].

This study found no significant difference in in-hospital complications, whether it was TKA, THA or pooled together. Previous studies about complications differ from each other. Kamath et al[1] did not found significant differences for complications between the simultaneous THA group and staged THA group either. Seung-Chan Kim et al[12] found a lower incidence of postoperative prosthetic-related complications in the simultaneous THA group. When it turns to TKA, several studies reported a lower rate of complications in the simultaneous TKA group[14,16,19,24,25]. Whereas some studies have reported a higher rate of specific complications, such as venous thromboembolism (VTE) [7] and myocardial infarction (MI)[26] for the patients older than 65years in the simultaneous TKA group. However, Sheth et al[27] considered 
that the differences in the baseline characteristics of the patients, surgeon's preference, and hospital characteristics may hamper the prior comparisons of complications between simultaneous TKA and staged TKA. They compared these two sub-groups by adjusting for these differences, founding no significant difference in complication rates between two subgroups. Since the data of our study came from a singer center, and the patient who underwent two procedures separated by the same surgeon was considered to have undergone staged bilateral TKA or THA, the inconsistency in-hospital characteristics, patient selection, surgeon skill, and surgeon preferences might be avoided. A meat-analysis[28] also proved no significant differences in complication rates. Moreover, studies that reported a higher rate of complication usually concentrated on certain complications, while our studies emphasized on the total number of in-hospital complications.

However, we also found a higher incidence of blood transfusion in the simultaneous groups compared with staged groups in all three groups. Sobh et al.[7], in a singer institution of 562 patients, reported a significantly increased rate of blood transfusion with simultaneous TKA and the same result was found in using a large Canadian data set[13]. In a series of bilateral total knee or hip arthroplasty, performed at a staged interval, would have more time for hematopoiesis to replenish blood loss because of the first surgery[29]. Most of the staged patients in our study waited more than 6 months between procedures. Kamath et al.[1] found no blood transfusion in either group, but a higher blood loss in the staged group for THA. Because of the limitation of data, we could not analyze the volume of blood transfused. Further study needs to clarify the relationship between blood loss volume and different ways of procedures. And different blood transfusion practices and standard of reporting in different hospitals and surgeons would influence the final result, the interpretation of these results should be cautious.

Owing to the limitation of data and the aim of our study, other clinical outcomes were not included. Relative to staged TKA, simultaneous TKA has been associated with higher 10-year prosthesis survival rates[2], longer quality-adjusted life years[16], the more economy of the physiotherapist's time, the less number of anesthetics and hospital admissions[25], lower frequency of having > 1 comorbid condition, lower frequency of knee infection[13]; insignificant differences regard to prosthetic failure, cardiac complications, and the rates of death[30]; no significant difference in Knee Society Score (KSS), Western Ontario and McMaster Universities Arthritis Index (WOMAC), and range of motion (ROM)[14]; and higher inhospital mortality, higher frequency of blood transfusions, higher frequency of transfer to a rehabilitation facility and higher rate of cardiac complications within 90 days[13].

Compared with staged bilateral procedures of THA, simultaneous THA has been associated with shorter operative and anesthetic times, less blood loss[1], lower leg-length discrepancy (LLD) and the percentage of patients who had an LLD >3 $\mathrm{mm}[12]$, and similar improvements in patient-reported outcomes at 1-year follow-up[31]. Most clinical outcomes were better for simultaneous TKA and THA, and they indeed have some advantages, compared with staged TKA and THA, such as less length of stay in the hospital, lower costs, no difference in complications which have been proved by this study. Accounting for a better surgical outcome, and relieving economic burden both for patients, families, and societies, we suggest that bilateral TKA and THA patients could be treated with a simultaneous TKA and THA rather than a staged TKA and THA. However, this procedure must be conducted very carefully, especially for elderly and high-risk patients.

Patients were older in the staged TKA and THA group than in the simultaneous TKA and THA group (Table1), which is consistent with previous studies[1,13]. Considering the operative risk, surgeons may prefer simultaneous procedures in younger and healthier patients, which might cause a selection bias and possibly result in better outcomes for simultaneous TKA and THA than staged TKA and THA. A recent study about the geriatric population reported that there was no association with any additional or significantly increased risk of morbidity or mortality compared to staged bilateral TKA[32]. Therefore, simultaneous TKA might be a safe and efficient choice for elderly people. While there need more researches to remove this age bias and prove the suitability for older people, particularly in THA patients. An adequately powered randomized trial, which could overcome the selection bias inherent in this retrospective study design, would be a good choice for further clarification of outcomes. 
These results may have important implications for the insurance department in the current health care environment. The cost of different surgeries for TKA and THA was different enough to warrant a separate classification for different procedures. Our results showed that staged bilateral TKA and THA have a greater financial cost than simultaneous bilateral TKA and THA, combined with the different clinical results of previous studies, suggesting that the two procedures should be classified separately for more accurate reimbursement. However, simultaneous bilateral TKA and THA and staged bilateral TKA and THA (calculate two surgical operations and reimbursed twice) are currently classified under the same Diagnosis Related Groups (DRGs), which means that they are reimbursed at the same level. Since medical insurance is the primary payer for patients in China[33], there needs reclassification of medical insurance items about these two procedures.

Limitations should be listed. Firstly, we investigated data from a single institution that performed a relatively high rate of TKA and THA, the universality of this study may be limited. However, this provided consistency in factors that might potentially affect clinical and financial outcomes, such as hospital characteristics, patient selection, surgeon skill, and surgeon preferences. Secondly, we did not analyze too many clinical outcomes because of the limitation of data, but our study aims to compare the medical expenditures and a fair number of previous studies have compared direct clinical outcomes. Thirdly, there might be a selection bias about the distribution of age, simultaneous procedures tending to be younger patients. But previous research has proved that simultaneous TKA might be a safe and efficient choice for elderly people. Further researches should include an adequately powered multi-center randomized trial, which could overcome the selection bias of this retrospective study design.

In spite of these limitations, the strengths of our study including all kinds of hospitalization costs and a number of patients in both TKA and THA patients, and pooled patients together to prove the results. To our knowledge, this is the first study to evaluate the direct costs between two procedures both for TKA and THA and the whole patients in the same cohort.

In summary, surgeons may wish to use our findings to help inform patients considering bilateral total arthroplasty. We prefer simultaneous bilateral TKA and THA for patients with stable disease when there are adequate indications for bilateral total arthroplasty. Further study needs to evaluate both the direct hospital costs and more clinical outcomes in the same cohort and an adequately powered randomized trial would be better.

\section{Conclusions}

The three groups of patients between two sub-groups had similar results, and no significant differences were found in inhospital complication. All kinds of medical expenses, with the exception of material fees, are lower for the simultaneous bilateral procedure, compared to a staged bilateral procedure whether it is TKA or THA or pooled groups. We also noted a significant difference in LOS, and the trends were the same as the costs. But simultaneous groups noted an increase in the risk of blood transfusion.

\section{Abbreviations}

TKA: total knee arthroplasty; THA: total hip arthroplasty; LOS: length of stay; OA: osteoarthritis; RA: rheumatoid arthritis; HIS: hospital information system; ICD-10

International Classification of Diseases,10th revision; TJA: total joint arthroplasty; CPI: Consumer Price Index; USD: US Dollars; IQR: interquartile ranges; IPR: patient rehabilitation; VTE: venous thromboembolism; MI: myocardial infarction; KSS: Knee Society Score; WOMAC: Western Ontario and McMaster Universities Arthritis Index; ROM: range of motion; LLD: lower leg-length discrepancy. 


\section{Declarations}

\section{Acknowledgment}

The authors would like to thank all the surgeons for giving permission to the use of their data.

\section{Funding}

This research did not receive any specific grant from funding agencies in the public, commercial, or not-for-profit sectors.

\section{Availability of data and materials}

The datasets supporting the conclusions of this article are included within the article/tables. The raw data can be requested from the corresponding author.

\section{Authors' contributions}

All authors have contributed to this study. JF H and $\mathrm{CH}$ conceived and designed this study; JF H wrote the drift of this paper; $\mathrm{CH}$ for assisting in the collation and arrangement of the data presented; $\mathrm{YZ} \mathrm{L}$ and $\mathrm{CZ}$ for writing assistance and proofreading the article; $\mathrm{JL}$ and $\mathrm{YW}$ for his guidance in statistical analysis in this study, and helped the interpretation of the data; CC L contributed to study coordination and was responsible for the English spelling and grammar; and $Y Z$ in charge of revision and guidance during the whole writing process of this thesis. All authors have participated in discussion, read and approved the final manuscript.

\section{Competing Interest}

The authors declare that they have no conflict of interest.

\section{Consent to publish}

Not Applicable

\section{Ethics approval and consent to participate}

This study was reviewed and approved by the Ethics Committee of the Affiliated Hospital of Qingdao University. All study participates, or their legal guardian, provided informed written consent prior to study enrollment.

\section{References}

1.Kamath AF, Monteiro EL, Spranger A, Impellizzeri F, Leunig M. Simultaneous versus staged bilateral direct anterior Total Hip Arthroplasty: Are early patient-centered outcomes equivalent? Acta Orthop Belg. 2016;82(3):497-508.

2.Lin AC, Chao E, Yang CM, Wen HC, Ma HL, Lu TC. Costs of staged versus simultaneous bilateral total knee arthroplasty: a population-based study of the Taiwanese National Health Insurance Database. J Orthop Surg Res. 2014;9:59. 
3.Ethgen O, Bruyere O, Richy F, Dardennes C, Reginster JY. Health-related quality of life in total hip and total knee arthroplasty. A qualitative and systematic review of the literature. J Bone Joint Surg Am. 2004;86(5):963-74.

4.Sloan M, Premkumar A, Sheth NP. Projected Volume of Primary Total Joint Arthroplasty in the U.S., 2014 to 2030. J Bone Joint Surg Am. 2018;100(17):1455-60.

5.Kurtz SM, Ong KL, Lau E, Bozic KJ. Impact of the economic downturn on total joint replacement demand in the United States: updated projections to 2021. J Bone Joint Surg Am. 2014;96(8):624-30.

6.Fu-xing Pei. The current status and future perspective of hip and knee arthroplasty in China[J]Chinese Journal of Bone and Joint. February 2012;1(01): V4-8 (In Chinese)

7.Sobh AH, Siljander MP, Mells AJ, Koueiter DM, Moore DD, Karadsheh MS. Cost Analysis, Complications, and Discharge Disposition Associated With Simultaneous vs Staged Bilateral Total Knee Arthroplasty. The Journal of arthroplasty. 2018;33(2):320-3.

8.Hustedt JW, Goltzer O, BohI DD, Fraser JF, Lara NJ, Spangehl MJ. Calculating the Cost and Risk of Comorbidities in Total Joint Arthroplasty in the United States. The Journal of arthroplasty. 2017;32(2):355-61.e1.

9.Elmallah RK, Chughtai M, Khlopas A, Bhowmik-Stoker M, Bozic KJ, Kurtz SM, et al. Determining Cost-Effectiveness of Total Hip and Knee Arthroplasty Using the Short Form-6D Utility Measure. The Journal of arthroplasty. 2017;32(2):351-4.

10.Peel TN, Cheng AC, Liew D, Buising KL, Lisik J, Carroll KA, et al. Direct hospital cost determinants following hip and knee arthroplasty. Arthritis Care Res (Hoboken). 2015;67(6):782-90.

11.Ma C, Jiang Y, Li Y, Zhang Y, Wang X, Ma S, et al. Medical expenditure for middle-aged and elderly in Beijing. BMC Health Serv Res. 2019;19(1):360.

12.Kim SC, Lim YW, Jo WL, Park DC, Lee JW, Kang WW, et al. Surgical accuracy, function, and quality of life of simultaneous versus staged bilateral Total hip Arthroplasty in patients with Osteonecrosis of the femoral head. BMC Musculoskelet Disord. 2017;18(1):266.

13.Bohm ER, Molodianovitsh K, Dragan A, Zhu N, Webster G, Masri B, et al. Outcomes of unilateral and bilateral total knee arthroplasty in 238,373 patients. Acta Orthop. 2016;87 Suppl 1:24-30.

14.Seol JH, Seon JK, Song EK. Comparison of postoperative complications and clinical outcomes between simultaneous and staged bilateral total knee arthroplasty. J Orthop Sci. 2016;21(6):766-9.

15.Courtney PM, Melnic CM, Alosh H, Shah RP, Nelson CL, Israelite CL. Is bilateral total knee arthroplasty staged at a oneweek interval safe? A matched case control study. The Journal of arthroplasty. 2014;29(10):1946-9.

16.Odum SM, Troyer JL, Kelly MP, Dedini RD, Bozic KJ. A cost-utility analysis comparing the cost-effectiveness of simultaneous and staged bilateral total knee arthroplasty. J Bone Joint Surg Am. 2013;95(16):1441-9.

17.Reuben JD, Meyers SJ, Cox DD, Elliott M, Watson M, Shim SD. Cost comparison between bilateral simultaneous, staged, and unilateral total joint arthroplasty. The Journal of arthroplasty. 1998;13(2):172-9.

18.Brotherton SL, Roberson JR, de Andrade JR, Fleming LL. Staged versus simultaneous bilateral total knee replacement. The Journal of arthroplasty. 1986;1(4):221-8.

19.Ritter MA, Meding JB. Bilateral simultaneous total knee arthroplasty. The Journal of arthroplasty. 1987;2(3):185-9. 
20.Raquel B, Javier M, Jesús C, Susana P, Sara Nistal J, Juan H, et al. Prolonged length of stay in hospitalized internal medicine patients. European Journal of Internal Medicine. 2015;26(10):772-5.

21.Doctoroff L, Hsu DJ, Mukamal KJ. Trends in prolonged hospitalizations in the United States from 2001 to 2012: a longitudinal cohort study. American Journal of Medicine. 2016;130(4):483.e1.

22.Shinjo D, Tachimori H, Sakurai K, Ohnuma T, Fujimori K, Fushimi K. Factors affecting prolonged length of stay in psychiatric patients in Japan: A retrospective observational study. Psychiatry \& Clinical Neurosciences. 2017;71(8).

23.Abdullah HR, Sim YE, Hao Y, Lin GY, Ghc L, Lamoureux EL, et al. Association between preoperative anaemia with length of hospital stay among patients undergoing primary total knee arthroplasty in Singapore: a single-centre retrospective study. Bmj Open. 2017;7(6):e016403.

24.Yoon HS, Han CD, Yang IH. Comparison of simultaneous bilateral and staged bilateral total knee arthroplasty in terms of perioperative complications. The Journal of arthroplasty. 2010;25(2):179-85.

25.Stanley D, Stockley I, Getty CJ. Simultaneous or staged bilateral total knee replacements in rheumatoid arthritis. A prospective study. J Bone Joint Surg Br. 1990;72(5):772-4.

26.Bolognesi MP, Watters TS, Attarian DE, Wellman SS, Setoguchi S. Simultaneous vs staged bilateral total knee arthroplasty among Medicare beneficiaries, 2000-2009. The Journal of arthroplasty. 2013;28(8 Suppl):87-91.

27.Sheth DS, Cafri G, Paxton EW, Namba RS. Bilateral Simultaneous vs Staged Total Knee Arthroplasty: A Comparison of Complications and Mortality. The Journal of arthroplasty. 2016;31(9 Suppl):212-6.

28. Hussain N, Chien T, Hussain F, Bookwala A, Simunovic N, Shetty V, et al. Simultaneous versus staged bilateral total knee arthroplasty: a meta-analysis evaluating mortality, peri-operative complications and infection rates. HSS journal: the musculoskeletal journal of Hospital for Special Surgery. 2013;9(1):50-9.

29.Fu D, Li G, Chen K, Zeng H, Zhang X, Cai Z. Comparison of clinical outcome between simultaneous-bilateral and stagedbilateral total knee arthroplasty: a systematic review of retrospective studies. The Journal of arthroplasty.

2013;28(7):1141-7.

30.Ritter MA, Harty LD, Davis KE, Meding JB, Berend M. Simultaneous bilateral, staged bilateral, and unilateral total knee arthroplasty. A survival analysis. J Bone Joint Surg Am. 2003;85(8):1532-7.

31.Degen RM, Nawabi DH, Fields KG, Wentzel CS, Kelly BT, Coleman SH. Simultaneous Versus Staged Bilateral Hip Arthroscopy in the Treatment of Femoroacetabular Impingement. Arthroscopy: the journal of arthroscopic \&amp; related surgery: official publication of the Arthroscopy Association of North America and the International Arthroscopy Association. 2016;32(7):1300-7.

32.Vaishya R, Vijay V, Mani KCK, Agarwal AK. Is simultaneous bilateral total knee arthroplasty safe in geriatric population? A retrospective cohort study with upto 9 years follow up. Journal of clinical orthopaedics and trauma. 2018;9(2):107-11.

33.Dou G, Wang Q, Ying X. Reducing the medical economic burden of health insurance in China: Achievements and challenges. Bioscience trends. 2018;12(3):215-9.

\section{Tables}

Table 1 Demographic and clinical characteristics of patients 


\begin{tabular}{|c|c|c|c|c|c|c|c|c|c|}
\hline \multirow{2}{*}{$\frac{\text { Groups }}{\text { Variables }}$} & \multicolumn{3}{|c|}{ Total knee arthroplasty } & \multicolumn{3}{|c|}{ Total hip arthroplasty } & \multicolumn{3}{|c|}{ Pooled groups } \\
\hline & $\begin{array}{l}\text { Simultaneous } \\
(\mathrm{n}=789)\end{array}$ & $\begin{array}{c}\text { Staged } \\
(\mathrm{n}=391)\end{array}$ & $\begin{array}{c}\mathrm{P} \\
\text { Value }\end{array}$ & $\begin{array}{l}\text { Simultaneous } \\
(\mathrm{n}=140)\end{array}$ & $\begin{array}{c}\text { Staged } \\
(\mathrm{n}=259)\end{array}$ & $\begin{array}{c}\mathrm{P} \\
\text { Value }\end{array}$ & $\begin{array}{l}\text { Simultaneous } \\
(\mathrm{n}=929)\end{array}$ & $\begin{array}{c}\text { Staged } \\
(\mathrm{n}=650)\end{array}$ & $\begin{array}{c}\mathrm{P} \\
\text { Value }\end{array}$ \\
\hline $\begin{array}{c}\text { age, } \\
\text { median(IQR) } \\
\text { years }^{\mathrm{a}}\end{array}$ & $64(60,69)$ & $68(63,73)$ & $<0.000$ & $53(47,60)$ & $58(49,64)$ & 0.001 & $63(58,68)$ & $64(58,70)$ & 0.031 \\
\hline 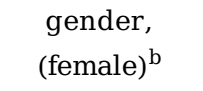 & $651(82.51 \%)$ & $314(80.31 \%)$ & 0.356 & $42(30.00 \%)$ & $85(32.82 \%)$ & 0.564 & $693(74.60 \%)$ & $399(61.38 \%)$ & $<0.000$ \\
\hline $\begin{array}{c}\text { complication, } \\
\text { yes }^{b}\end{array}$ & $20(2.53 \%)$ & $11(2.81 \%)$ & 0.778 & $4(2.86 \%)$ & $4(1.54 \%)$ & 0.372 & $24(2.58 \%)$ & $15(2.31 \%)$ & 0.728 \\
\hline $\begin{array}{c}\text { LOS, } \\
\text { median(IQR) } \\
\text { a }\end{array}$ & $9(8,11)$ & $18(15,20)$ & $<0.000$ & $10(8,11)$ & $16(14,18)$ & $<0.000$ & $9(8,11)$ & $17(15,19)$ & $<0.000$ \\
\hline $\begin{array}{c}\text { blood } \\
\text { transfusion, } \\
\text { yes }^{\mathrm{b}}\end{array}$ & $302(38.28 \%)$ & $32(8.18 \%)$ & $<0.000$ & $51(36.43 \%)$ & $36(13.90 \%)$ & $<0.000$ & $353(37.99 \%)$ & $68(10.46 \%)$ & $<0.000$ \\
\hline
\end{tabular}

IQRDinterquartile range; LOS, length of stay.

a Continuous data were presented as median (IQR) and compared by Mann-Whitney U test; $\quad$ b categorical variables were expressed by counts and percentages and compared by the Chi-square test or the Fisher's exact test, as appropriate.

Due to technical limitations, table 2 is only available as a download in the supplemental files section.

\section{Supplementary Files}

This is a list of supplementary files associated with this preprint. Click to download.

- supplement1.doc

- supplement2.pdf 